\title{
Estandarización de ELISA para el diagnóstico de fasciolosis bovina, ovina y humana
}

\author{
Standardization of ELISA for the diagnosis of \\ bovine, ovine and human fasciolosis
}

\author{
Raúl Sierra-Balcárcel ${ }^{1}$; Ruth-Aralí Martínez-Vega ${ }^{1}$; Reynaldo Gutiérrez-Marín²; \\ Cecilia-Dolores Colmenares ${ }^{3}$; Nelson Uribe-Delgado ${ }^{1}$
}

Forma de citar: Sierra Barcácel R, Martínez Vegal RA, Gutiérrez Marín R, Colmenares CD, Uribe Delgado N. Estandarización de ELISA para el diagnóstico de fasciolosis bovina, ovina y humana. Rev Univ Ind Santander Salud. 2017; 49(4): 549-556. doi: http://dx.doi.org/10.18273/revsal.v49n4-2017004 (c) (1)

\section{Resumen}

Introducción: La fasciolosis representa un grave problema para el sector pecuario y la salud humana en muchos países. En Colombia, afecta principalmente al ganado bovino y ovino, con escasos reportes en humanos. Objetivo: Estandarizar un Ensayo de Inmunoadsorción Enzimática para bovinos, ovinos y humanos como herramienta de tamizaje de fasciolosis. Metodología: Se utilizaron 50 muestras de bovinos, 50 de ovinos y 39 de humanos con diagnóstico de fasciolosis confirmado por serología y examen de materia fecal. Se utilizaron cuatro presentaciones del antígeno excretor-secretor de Fasciola hepatica. Se calcularon la sensibilidad, la especificidad y la concordancia. Resultados: La sensibilidad del ELISA en humanos, ovinos y bovinos fue de $100 \%$ y la especificidad fue de 97\%, 85,2\% y 96,2\%, respectivamente. El coeficiente Kappa de Cohen fue superior a 0,8 en las tres especies. Conclusiones: La prueba de ELISA estandarizada para el diagnóstico de la fasciolosis humana, ovina y bovina demostró una excelente sensibilidad y buena especificidad. Se propone realizar la validación a mayor escala para su posterior uso como herramienta en el tamizaje de esta parasitosis.

Palabras clave: Ensayo de Inmunoadsorción Enzimática; Diagnóstico; Fascioliasis; Fasciola hepatica; Estándares de Referencia; Humanos; Ovinos; Bovinos.

\begin{abstract}
Introduction: Fascioliasis represents a serious problem for livestock production and human health in the world. In Colombia, it mainly affects bovine and ovine cattle, with few cases reported in humans. Objective: To standardize an Enzyme-Linked ImmunoSorbent test (ELISA) in bovine, ovine, and human individuals as a screening tool of fascioliasis. Methods: 50 bovine, 50 ovine and 39 human samples with fascioliasis confirmed by serology and stool test were used. Sensitivity, specificity and concordance were calculated. Results: Sensitivity of the ELISA in humans, bovine and ovine cattle was $100 \%$ and specificity was $97 \%, 85.2 \%$ and $96.2 \%$, respectively. Cohen's Kappa coefficient was $>0.8$ in all groups. Conclusions: Standardized ELISA test for bovine, ovine and human fascioliasis exhibited an excellent sensitivity and good specificity. It is proposed to carry out a large-scale validation for its ulterior use as a screening tool for the diagnosis of this parasitic infection.
\end{abstract}

Keywords: Enzyme-Linked Immunosorbent Assay; Diagnosis; Fascioliasis; Fasciola hepatica; Standardization; Humans; Sheep; Cattle.

1. Universidad Industrial de Santander. Bucaramanga, Colombia.

2. Universidad de Pamplona. Pamplona, Norte de Santander.

3. Universidad Central de Venezuela. Caracas, Venezuela.

Correspondencia: Nelson Uribe Delgado. Dirección: Carrea 32 29-31. Correo electrónico: nelurdel@uis.edu.co. Teléfono: +57 3204456726 


\section{Introducción}

La fasciolosis es una zoonosis cosmopolita, considerada como una de las enfermedades parasitarias más importantes de los rumiantes domésticos, que además afecta a gran cantidad de animales herbívoros y omnívoros, ocasionalmente al hombre y raramente aves $^{1}$. Se caracteriza por un proceso inflamatorio del hígado y el conducto biliar, frecuentemente crónico y relacionado con trastornos de asimilación de los nutrientes. El agente etiológico en Colombia y otros países de América, África y Europa es Fasciola hepatica, mientras que Fasciola gigantica lo es en países de Asia $^{2}$.

La distribución geográfica del parásito depende de la presencia de los moluscos hospedadores intermediarios pertenecientes a la familia Lymnaeidae, los cuales han sido reportados en los más diversos pisos térmicos, entre 0 y más de $4.000 \mathrm{msnm}$. Así mismo, F. hepatica es el único parásito capaz de originar zonas hiperendémicas humanas desde el nivel del mar, como en la provincia de Gilán en Irán, hasta altitudes superiores a 3.800 msnm en altiplanos y valles de Bolivia y Perú. No hay otro helminto que presente tan amplia distribución altitudinal y latitudinal como este trematodo ${ }^{3}$.

La fasciolosis ocasiona un grave problema socioeconómico para el sector pecuario mundial, con cifras superiores a 600 millones de animales infectados y presentándose en algunas regiones prevalencias entre $25 \%$ y $100 \%$ en ganado vacuno y búfalos. Representa un significativo impacto económico principalmente en ovinos y bovinos con pérdidas anuales calculadas en US 3.000 millones $^{4}$. En Colombia en 1996 se estimaron las pérdidas en $\$ 12.500$ millones $^{5}$. Sin embargo, este valor debe ser nuevamente calculado ya que han sido descritas nuevas zonas endémicas de fasciolosis después de ese año.

Esta parasitosis en Colombia y otros países recibe mayor atención en el sector pecuario por la alta prevalencia e impacto económico que presenta, si se compara con el interés que despierta en el ámbito de la salud humana. No obstante, en las dos últimas décadas se ha calificado como emergente y reemergente en humanos, presentándose en los cinco continentes, en diferentes latitudes y altitudes, por lo que la Organización Mundial de la Salud (OMS) ha lanzado una iniciativa de control, considerándola un problema de salud pública mundial. La infección en humanos se ha incrementado en casos y número de países afectados. En 1995 la OMS estimó en 2,4 millones las personas infectadas y 180 millones las que están en riesgo de adquirirla; sin embargo, otras fuentes plantean cifras superiores a 17 millones de personas las que sufren la enfermedad ${ }^{6,7}$. Estos datos pueden no ser reales ya que no se cuenta con información actualizada que dimensione con precisión la prevalencia mundial.

En algunas regiones de economía campesina la concurrencia de diferentes especies de explotación pecuaria como bovinos y ovinos es frecuente, y se constituye en un factor que facilita la endemicidad de la parasitosis animal. Así pues, se ha demostrado que en la contaminación continua de los pastos, los ovinos con infección crónica juegan un papel importante en el mantenimiento y diseminación del parásito, ya que un solo ovino puede eliminar entre 2,5 a 3,5 millones de huevos en un día, aumentando la probabilidad de infección de otros herbívoros susceptibles y de los humanos que viven en estas regiones ${ }^{7,8}$. A pesar de lo anterior, los pequeños y medianos productores de los países del tercer mundo no utilizan las herramientas diagnósticas como parte de las buenas prácticas en el manejo de los animales debido a cuestiones de tipo cultural, costos o dificultad para acceder a ellas ${ }^{9}$.

Considerando el problema que esta parasitosis significa para la salud humana y animal, es esencial contar con técnicas de laboratorio que ofrezcan buena sensibilidad-especificidad, bajo costo, alto rendimiento y reproducibilidad.

El diagnóstico de rutina de la fasciolosis animal se hace utilizando el examen coprológico, principalmente mediante la técnica de Dennis para la búsqueda de huevos, lo cual es un hallazgo de gran utilidad pues informa de una infección o enfermedad actual. Sin embargo, estas técnicas presentan algunas desventajas como la baja sensibilidad que depende, entre otras razones, del momento de inicio y la intermitencia en la ovoposición y de la carga parasitaria. Además, la observación de los huevos requiere adiestramiento para su correcta identificación y para no confundirlos con los huevos de paramphistomidos. Una dificultad adicional, es que sólo se encuentran huevos después de 12 semanas post-infección, cuando el daño hepático es avanzado.

La respuesta inmune en la fase de invasión y migración del parásito en los hospedadores definitivos es principalmente de tipo Th2, con altos títulos de $\operatorname{IgG}$ detectables desde la segunda semana post-infección $\mathrm{y}$ por largo tiempo, sirviendo como marcador de la infección aguda y crónica y excelente isotipo a determinar mediante ELISA ${ }^{10-12}$. 
Estas son algunas de las razones por lo que diversos grupos de investigación en el mundo han estandarizado esta técnica para el diagnóstico de la fasciolosis en humanos y animales. Para ello se vienen implementando diversas variantes de la metodología que buscan el mayor rendimiento de la prueba, para la cual se ha utilizado una diversidad de antígenos como: extractos somáticos totales, metabólicos, recombinantes y fracciones purificadas ${ }^{13-21}$. Otra metodología empleada en el diagnóstico de la fasciolosis es el Western blot o inmunoelectrotransferencia (EITB), que permite aumentar la especificidad además de evaluar las proteínas inmunodominates ${ }^{15-19}$ que pueden ser empleadas como marcadores específicos en pruebas serológicas ${ }^{6}$; sin embargo, este método no es empleado en la mayoría de laboratorios en los cuales se realiza diagnóstico de la patología, ya que no cuenta con estándares que permitan su rápida masificación.

El antígeno excretor secretor de Fasciola hepatica (AgE/ $\mathrm{SFh}$ ) juega un importante papel en la estimulación de la respuesta de anticuerpos detectada desde la segunda semana postinfección y antes de la aparición de la sintomatología y de los hallazgos parasitológicos ${ }^{10,11}$. Este antígeno ha sido utilizado en diferentes presentaciones y técnicas de inmunodiagnóstico con buenos resultados de sensibilidad (S) y especificidad (E), valores que oscilan entre el 70 y $100 \%$ para las dos probabilidades diagnósticas ${ }^{14-16,19-21}$.

Como alternativa a la baja sensibilidad del examen coprológico, y respondiendo a la necesidad de contar con una técnica de diagnóstico versátil, este artículo presenta los resultados de la estandarización de un ELISA para fasciolosis en bovinos, ovinos y humanos utilizando el antígeno AgE/SFh en las condiciones locales, comparándola con una técnica desarrollada en la Universidad Central de Venezuela ${ }^{21}$. La estandarización de la misma se efectuó con el propósito de que sirva como herramienta de tamizaje a nivel nacional.

\section{Materiales y métodos}

Obtención del Antígeno. Para obtener el antígeno se recolectaron vermes de hígados de bovinos infectados con $F$. hepatica proporcionados por la planta de sacrificio "El Vijagual" de la ciudad de Bucaramanga. Los parásitos fueron recogidos en solución salina $0,9 \%$ y mantenidos a $37^{\circ} \mathrm{C}$ durante el traslado al laboratorio. El AgE/SFh se obtuvo incubando los parásitos individualmente en $1 \mathrm{~mL}$ de medio RPMI 1640 durante 30 horas con recambio cada seis horas, y bajo una un atmósfera de $5 \%$ de $\mathrm{CO}_{2}$. Una vez extraído el medio, se añadió un coctel de inhibidores de proteasas (PMFS $1 \mathrm{mM}$, EDTA $0,5 \mathrm{M}$ ) y penicilina $(10.000 \mathrm{UI} / \mathrm{mL})$; posteriormente se liofilizó y reconstituyó en PBS. A este antígeno se llamó Preparado 1 (P1). Con el objetivo de obtener y probar otros preparados del antígeno se hicieron dos modificaciones: La primera consistió en adicionar Ácido Tricloro-Acético (20\%) al producto reconstituido después de ser liofilizado (P2) y en la segunda modificación, el producto reconstituido se pasó por membranas Amicon ${ }^{\circledR}$ Y 100 y Y50 para obtener fracciones proteicas de diferente peso molecular. A los productos retenidos se le denominó R100 y R50, respectivamente. Los cuatro antígenos fueron analizados utilizando PAGE al 12\% para comprobar la integridad de las proteínas. La concentración de proteínas se calculó utilizando el método de Bradford (Quickstart, Biora), realizando la lectura a $595 \mathrm{~nm}$ en un equipo iMark microplate reader de Biorad ${ }^{\circledR}$.

La diferencia entre las dos pruebas serológicas (colombiana y venezolana) radica fundamentalmente en la preparación del $\mathrm{AgE} / \mathrm{Sfh}$. En la técnica colombiana se hizo recambio de medio cada seis horas para seleccionar el momento en que se encontrara la mayor concentración de proteínas. Además, se corrieron tres protocolos distintos luego de la liofilización respectiva, dando finalmente cuatro tipos de antígenos a probar.

Muestras. Los sueros utilizados para la estandarización fueron analizados para establecer si eran positivos o negativos a fasciolosis mediante ELISA en la UCV. Se realizó también el examen coprológico, por la técnica de Dennis modificada para la búsqueda de huevos de $F$. hepatica, en el laboratorio de parasitología de la Universidad Industrial de Santander (UIS), con excepción de algunos que no fue posible recuperar la muestra.

Sueros humanos. Como controles positivos de fasciolosis humana se incluyeron seis sueros reactivos suministrados por el Laboratorio de Inmunología de la UCV y 33 negativos provenientes del área urbana de la ciudad de Bucaramanga, Santander, Colombia.

Sueros bovinos y ovinos. Se tomaron 24 sueros positivos de bovinos y 23 de ovinos en zona endémica de fasciolosis de la Provincia de García Rovira en Santander, y 26 negativos de bovinos y 27 de ovinos en Zapatoca, Santander, Colombia. Los sueros fueron analizados mediante ELISA en el Laboratorio de Inmunología de la UCV. 
Sueros para estudio de reacciones cruzadas. Para este componente del estudio fueron evaluados 22 bovinos, 21 ovinos y 27 humanos, negativos por coprología y serología a $F$. hepatica y con presencia de huevos y/o larvas de otros parásitos en las heces. Los sueros humanos fueron obsequiados por el Centro de Investigación de Enfermedades Tropicales de la UIS y analizados mediante ELISA en el Laboratorio de Inmunología de la UCV. Las muestras fecales se analizaron en el Laboratorio de Parasitología de la UIS.

Los sueros humanos fueron obtenidos de personas diagnosticadas por coprología o serología a Tenia sp., Ascaris lumbricoides, Strongyloides stercolaris, Uncinarias, Trichuris trichiura, Leishmania sp., Trypanosoma cruzi, Entamoeba histolytica, VIH, Toxocara sp., Toxoplasma gondii e Isospora belli. Los sueros de bovinos y ovinos fueron obtenidos de animales positivos a Trichostrongylus sp., Cooperia sp., Eimeria sp., Oesophophagostomun sp., Toxocara sp., Haemonchus sp., Moniezia sp., Ostertagia sp., Paramphistomum sp., Nematodiurus sp., y Bonostomum sp.

Estandarización del ELISA. La concentración inicial de proteína de los antígenos fue $7,7 \mu \mathrm{g} / \mu \mathrm{L}$ para $\mathrm{P} 1 ; 5,1$ $\mu \mathrm{g} / \mu \mathrm{L}$ para $\mathrm{P} 2 ; 6,9 \mu \mathrm{g} / \mu \mathrm{L}$ para $\mathrm{R} 50$ y $10,5 \mu \mathrm{g} / \mu \mathrm{L}$ para R100. Las concentraciones de cada uno de los cuatro antígenos para cruzar con los otros parámetros en la estandarización del ELISA fueron 1, 1,5, 2,5 y $5 \mu \mathrm{g}$ / $\mathrm{mL}$, procedimiento que se realizó en la misma placa (Greiner bio-one ${ }^{\circledR}$. Apogent $®$. Fondo plano). Como bloqueadores se ensayaron leche descremada al 1 y $5 \%$, leche de soya al 1 y $5 \%$ y gelatina al 0,2 y $0,5 \%$. Todos los ensayos se realizaron con solución bloqueadora.

Las diluciones utilizadas de los sueros (controles y desconocidos) fueron: $1 / 100,1 / 200$ y $1 / 500$ para humanos; 1/200, 1/500, $1 / 800$ para bovinos y $1 / 100,1 / 200,1 / 500$ y $1 / 1000$ para ovinos. Las diluciones probadas del conjugado anti-IgG fueron $1: 10.000 / 20.000 / 30.000$ y 50.000 para ovinos-bovinos y $1: 30.000 / 40.000$ y 50.000 para humanos. El substrato utilizado fue ortofenil-dietanolamina (OPD) probado en concentraciones de 2, 4, 6, 8, 10 y $20 \mathrm{mg} /$ placa.

Procedimiento. El ensayo inmunoenzimático se realizó de acuerdo a Voller, et al. ${ }^{22}$ La sensibilización de los pozos se hizo con $100 \mu \mathrm{L}$ del antígeno en buffer carbonato $(\mathrm{pH} 9,6)$ por 18 horas a $4^{\circ} \mathrm{C}$. Se efectuaron tres lavados con PBS-Tweem 20 por dos minutos y luego incubación durante una hora a $37^{\circ} \mathrm{C}$ de $100 \mu \mathrm{L}$ de los sueros diluidos; se realizaron nuevos ciclos de lavados anteriores y se adicionó $100 \mu \mathrm{L} /$ pozo de conjugado incubando las placas a $37^{\circ} \mathrm{C}$ por una hora.
Se retiró el exceso de conjugado con nuevo ciclo de lavados. Se adicionó $100 \mu \mathrm{L}$ por pozo de la mezcla de orto-fenil-dietanolamina (OPD) disuelta en ácido cítrico y fosfato disódico a pH 5,0 y $10 \mu \mathrm{L}$ de peróxido de hidrógeno. Las placas se incubaron ocho minutos, en oscuridad, a $21^{\circ} \mathrm{C}$ y la reacción se detuvo con $\mathrm{H}_{2} \mathrm{SO}_{4}$ y $2 \mathrm{~N}$. Las lecturas se realizaron en un equipo iMark Microplate Absorbance Reader de Biorad® a $490 \mathrm{~nm}$. Los puntos de corte fueron obtenidos del promedio de las absorbancias de todos los sueros seleccionados para la estandarización que fueron negativos, más tres desviaciones estándar.

Análisis estadístico. Para los cálculos de la sensibilidad, especificidad y el cofeciente Kappa de Cohen se empleó el paquete estadístico STATA 12.0. Se consideró como patrón de oro, el ELISA realizado en el laboratorio de inmunología de la UCV, ya que se conocía a cabalidad la procedencia de las muestras y se demostró su resultado respectivamente.

\section{Resultados}

Antígenos. En la electroforesis realizada a los cuatro antígenos se observaron bandas de diferente peso molecular, siendo común en todos ellos los componentes proteicos entre 28 y $30 \mathrm{kDa}$ (Figura 1).

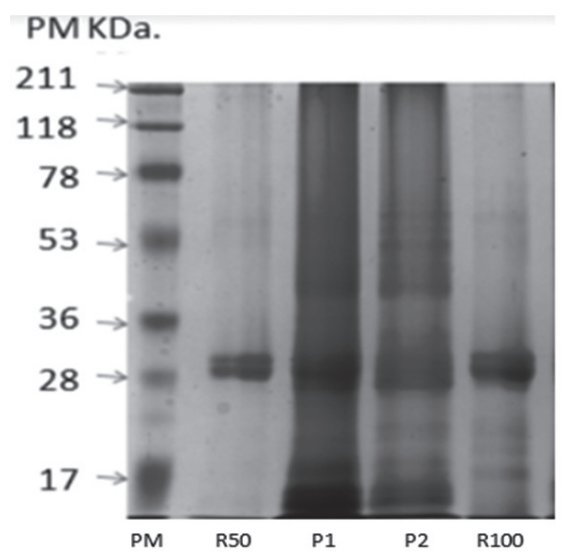

Figura 1. Electroforesis de los cuatro antígenos probados en la estandarización

La discriminación entre controles positivos y negativos fue similar con los cuatro antígenos en las concentraciones probadas; sin embargo, se seleccionó para la estandarización el R50, con una concentración óptima de $1,5 \mu \mathrm{g} / \mathrm{mL}$, porque las absorbancias observadas fueron más altas dentro del grupo de los positivos.

Estandarización del ELISA. Las diluciones óptimas de los sueros fueron 1:200 para humanos y ovinos y 1:500 para bovinos; el bloqueador que dio mejores 
resultados fue la leche descremada al 5\% para las tres especies; las diluciones óptimas del conjugado fueron 1:50.000 para humanos y 1:20.000 para los animales (Tabla 1) y la concentración del sustrato escogida fue la de $10 \mathrm{mg} /$ placa con un tiempo de reacción de 8 minutos a $22^{\circ} \mathrm{C}$ y bloqueo de la misma con $50 \mu \mathrm{L}$ de ácido sulfúrico $2 \mathrm{~N}$ por pozo.

Tabla 1. Discriminación entre controles positivos y negativos utilizando diferentes diluciones de Anti IgG

\begin{tabular}{cccc}
\hline Anti IgG & Ovinos & Bovinos & Humanos \\
\hline $1: 10.000$ & ++ & ++ & - \\
$1: 20.000$ & +++ & +++ & - \\
$1: 30.000$ & ++ & ++ & + \\
$1: 40.000$ & - & - & ++ \\
$1: 50.000$ & + & + & +++ \\
\hline
\end{tabular}

- No hay discriminación de los controles positivos y negativos.

+ Poca discriminación de los controles positivos y negativos.

++ Moderada discriminación de los controles positivos y negativos.

+++ Alta discriminación de los controles positivos y negativos.

Determinación del punto de corte, sensibilidad y especificidad. Para humanos el punto de corte fue de 0,15; al comparar la prueba realizada en Colombia con la prueba realizada en Venezuela, se observó una sensibilidad de 100\% (IC95\% 54,1 - 100) y una especificidad de 97\% (IC95\% 84,2 - 99,9). Para ovinos el punto de corte fue de 0,18 , la prueba colombiana tuvo una sensibilidad de 100\% (IC95\% 85,2 - 100) y una especificidad de $85,2 \%$ (IC95\% 66,3 - 95,8). Para bovinos el punto de corte fue de 0,28 y al comparar las pruebas se observó una sensibilidad de 100\% (IC95\% $85,8-100$ ) y una especificidad de $96,2 \%$ (IC95\% 80,4 -99,9), respectivamente. El porcentaje de concordancia entre las pruebas fue alto para las tres poblaciones, con Kappa mayor a 0,8 (Tabla 2).

Tabla 2. Comparación de los resultados obtenidos por ELISA realizado en Colombia y por ELISA hecho en Venezuela.

\begin{tabular}{ccccccc}
\hline Población & $V^{a}$ & $V^{b}$ & $F^{c}$ & $F^{d}$ & $\%$ de Acuerdo & Kappa \\
\hline Humanos & $6 / 6$ & $32 / 33$ & 1 & 0 & 97,44 & 0,9078 \\
Ovinos & $23 / 23$ & $23 / 27$ & 4 & 0 & 92 & 0,8410 \\
Bovinos & $24 / 24$ & $25 / 26$ & 1 & 0 & 98 & 0,96 \\
\hline
\end{tabular}

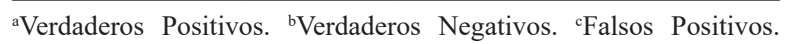
${ }^{\mathrm{d}}$ Falsos Negativos.

No se detectó ninguna reacción cruzada con los sueros positivos para los diferentes patógenos evaluados en las pruebas para bovinos, ovinos y humanos; los valores de absorbancia de estos sueros estuvieron entre $0,03-0,11$; 0,05-0,259 y 0,027-0,141, respectivamente (Figura 2).

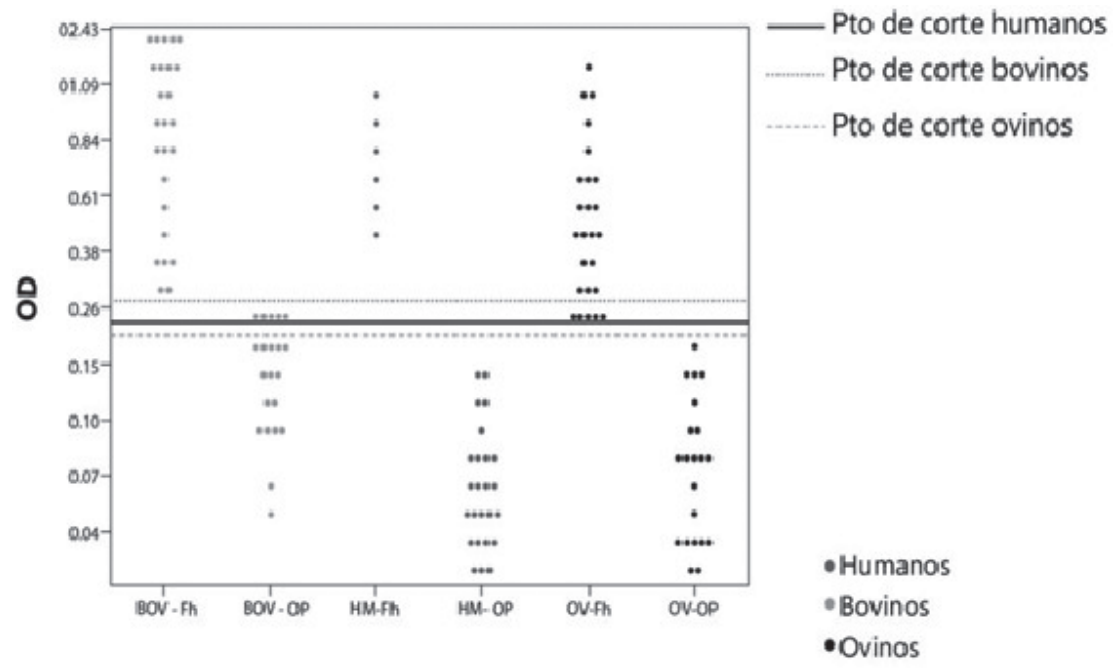

Figura 2. Densidad óptica del ELISA de los sueros de las tres especies con fasciolosis (FH) y después de sueros con otras parasitosis (OP).

\section{Discusión}

El uso del ELISA como prueba de tamizaje en fasciolosis ha pasado por un arduo debate, resultado de diversos trabajos experimentales iniciados en 1978 y que hoy posicionan esta técnica en un lugar destacado frente a otras herramientas diagnósticas. Uno de los elementos de controversia ha sido la capacidad discriminatoria de la técnica, que algunos autores relacionan directamente con el antígeno utilizado ${ }^{23,24}$.

Diversas preparaciones del antígeno se han probado para la estandarización del ELISA: antígenos somáticos, excretor-secretor, o fracciones de estos. Sin embargo, se 
ha dedicado especial atención (lo demuestra el número de trabajos realizados), a la utilización del AE/SFh completo y sus fracciones, pues se ha demostrado mejor rendimiento con este antígeno, lo que está en relación directa con la evidencia de que los productos metabólicos son responsables de una fuerte y temprana respuesta de anticuerpos en los hospedadores, permitiendo diagnosticar infecciones cuando aún no hay daño hepático ${ }^{15-21}$.

Los cuatro preparados del antígeno excretor-secretor de F. hepatica (AE/SFh), utilizados en el presente trabajo, no mostraron diferencias importantes en su capacidad discriminatoria entre controles positivos y negativos al realizar las pruebas preliminares en los ensayos de estandarización del ELISA para bovinos, ovinos y humanos. Sin embargo, la fracción R50 fue seleccionada como óptima debido a los valores superiores de las absorbancias que arrojó en el grupo de los positivos y porque ha sido reportado como un excelente antígeno por varios investigadores ${ }^{21}$, quienes encontraron fracciones que ofrecen alta sensibilidad y especificidad en ELISA y Western blot. En este último, las proteínas de 9, 14 y $27 \mathrm{kDa}$ de la fracción R50 son reconocidas por el 90\% al $100 \%$ de los sueros positivos y ninguno de los negativos a fasciolosis, ni los sueros positivos para otras helmintiosis, incluida la paragonimiosis. Estas evidencias posicionan a R50 como un excelente antígeno para hacer diagnóstico masivo en poblaciones humanas, previo a la prueba confirmatoria con las fracciones específicas identificadas por Western blot ${ }^{21}$.

La sensibilidad y la especificidad observadas en el presente trabajo muestran un excelente poder discriminatorio de las tres pruebas estandarizadas teniendo en cuenta las ventajas de esta metodología ${ }^{22}$. Los parámetros hallados en el presente trabajo muestran una sensibilidad para ovinos, bovinos y humanos de $100 \%$ y una especificidad entre $85,2 \%$ y $97 \%$, similares a los encontrados por otros investigadores que oscilan entre 90 y $100 \%$ para las dos características cuando se usa ELISA con el mismo antígeno o fracciones nativas o recombinantes de él, o mediante ELISA para detectar coproantígenos $^{17-21,24-27}$.

No se observaron reacciones cruzadas con los sueros probados lo cual diverge de otras investigaciones que han reportado valores hasta $30 \%$ de falsos positivos ${ }^{27-29}$ cuando se usa el AE/SFh total. A pesar de ello, los autores refieren que ello puede deberse a la no utilización de una fracción purificada. Particularmente, el trabajo de Colmenares, et al. ${ }^{21}$ muestra reactividad cruzada en ELISA y la existencia en Western blot de varias fracciones del R50, especialmente la de 75
$\mathrm{kDa}$, que son reconocidas por sueros de personas con paragonimiosis, hidatidosis, anquilostomiosis $\mathrm{y}$ ascaridiosis, lo cual evidencia la necesidad de realizar una prueba de Western blot con la fracción R50 obtenida en el presente trabajo.

Así mismo, la sensibilidad y la especificidad mostradas por el ELISA estandarizado en el Laboratorio Central de la Universidad Industrial de Santander para el diagnóstico de fasciolosis bovina, ovina y humana permiten recomendarla para su validación en estudios con mayor tamaño de muestra con el fin de poder utilizarla en el futuro como herramienta de tamizaje.

\section{Agradecimientos}

El presente trabajo contiene parte de los resultados obtenidos en el Proyecto de investigación "Estudio de Fasciolosis ovina y bovina en las veredas Carobobo, Anca, Listara, Cairasco, Corralfalso y Mortiño de la Provincia de García Rovira, Santander del Sur; financiado por Colciencias código 110252128902. RC No. 5592011. Convocatoria 521 de 2010. Programa Nacional de Ciencia, Tecnología e Innovación Agropecuaria.

A la sección de Inmunología del Instituto de Medicina Tropical, de la Universidad Central de Venezuela por su valiosa colaboración expresada en capacitación, diagnóstico de referencia y permanente asesoría.

\section{Consideraciones éticas}

El proyecto fue avalado por el Comité de Ética en Investigación Científica de la UIS, según consta en el Acta No. 19 de 2012.

\section{Conflicto de intereses}

Los autores declaramos no tener conflicto de intereses con ninguna institución o persona en la realización del presente estudio.

\section{Referencias}

1. Hurtrez-Boussès S, Meunier C, Durand P, Renaud F. Dynamics of hostparasite interactions: the example of population biology of the liver fluke (Fasciola hepatica). Microbes Infect. 2001; 3(10): 841-849. DOI: 10.1016/S1286-4579(01)01442-3.

2. Le TH, Van De N, Agatsuma T, Thi Nguyen TH, Nguyen QD, McManus DP, et al. Human fascioliasis and the presence of hybrid/introgressed 
forms of Fasciola hepatica and Fasciola gigantica in Vietnam. Int J Parasitol. 2008; 38(6): 725-730. DOI: 10.1016/j.ijpara.2007.10.003.

3. Mas-Coma S, Bargues MD, Valero MA, Fuentes MV. Adaptation capacities of Fasciola hepatica and their relationships with human fasciolosis: from below sea level up to the very high altitude. En: Combes C, Jourdane J. Taxonomy, ecology and evolution of metazoan parasites. Perpignan: Perpignan University Press; 2003: p.81-123.

4. Cwiklinski K, O’Neill SM, Donnelly S, Dalton JP. A prospective view of animal and human Fasciolosis. Parasite Immunol. 2016; 38(9): 558-568. DOI: 10.1111/pim.12343.

5. Benavides E. Diseño de planes racionales de control de parásitos internos de los rumiantes con base en los resultados de investigaciones sobre su dinámica poblacional. Rev Corpoica. 1996; 2: 79-88.

6. Morales A, Espino AM. Evaluation and characterization of Fasciola hepatica tegument protein extract for serodiagnosis of human fascioliasis. Clin Vaccine Immunol. 2012; 19(11): 1870-1878. DOI: 10.1128/CVI.00487-12.

7. Mas-Coma, S. Human fascioliasis. En: Cotruvo JA, Dufour A, Rees G, Bartram J, Carr R, Cliver DO, editors. Waterborne Zoonoses: Identification, causes and control. London: IWA Publishing; 2004: p.305-322.

8. Uribe N, García C. Fasciolosis, zoonosis emergente y reemergente vista desde una dimensión ambiental. Vitae. 2013; 56: 1-10.

9. Mas-Coma S. Epidemiology of fascioliasis in human endemic areas. J Helminthol. 2005; 79 (3): 207-216. DOI:10.1079/JOH2005296.

10. Mas-Coma S. Human fascoliasis: Epidemiological patterns in human endemic areas of South America, Africa and Asia. South Asian J Trop Med Public Health. 2004; 35(S1): 1-11.

11. Gupta SC. Fasciolosis in man and animals: an overview retrospect to historical perspective. Acts del XXIV national congress of veterinary parasitology \& national symposium on "towards food security through sustainable animal production and integrated parasite management"; 2014 Feb 5-7; Thrissur, India. College of veterinary $\&$ animal sciences; 2014. pp. 164-174.

12. Shafiei R, Sarkari B, Sadjjadi SM. Performance of a $27 \mathrm{kDa}$ Fasciola hepatica Antigen in the Diagnosis of Human Fascioliasis. J Lab Physicians. 2015; 7(1): 17-20. DOI: 10.4103/0974-2727.154781.

13. Carnevale S, Rodríguez MI, Guarnera EA, Carmona C, Tanos T, Angel SO. Immunodiagnosis of fasciolosis using recombinant procathepsin L cystein proteinase. Diagn Microbiol Infect Dis. 2001; 41(12): 43-49. DOI: 10.1016/S0732-8893(01)00288-7.

14. El Ridi R, Salah M, Wagih A, William H, Tallima $\mathrm{H}$, El Shafie $\mathrm{MH}$, et al. Fasciola gigantica excretory-secretory products for immunodiagnosis and prevention of sheep fasciolosis. Vet Parasitol. 2007; 149(3-4): 219-228. DOI: 10.1016/j. vetpar.2007.08.024.

15. Escalante H, Davelois K, Ortiz P, Rodríguez H, Díaz E, Jara C. Western blot technique standardization of the diagnosis of human fasciolosis using Fasciola hepatica excreted-secreted antigens. Rev Peru Med Exp Salud Publica. 2011; 28(3): 454-461. DOI: 10.1590/S1726-46342011000300008.

16. Rivera-Marrero CA, Santiago N, Hillyer GV. Evaluation of immunodiagnostic antigens in the excretory-secretory products of Fasciola hepatica. J Parasitol. 1988; 74(4): 646-652. DOI: $10.2307 / 3282184$.

17. Espino AM, Borges A, Duménigo BE. Coproantígenos de Fasciola hepatica de posible utilidad en el diagnóstico de la fasciolosis. Rev Panam Salud Pública. 2000; 7(4): 225-231. DOI: 10.1590/S1020-49892000000400003.

18. Kim K, Yang HJ, Chung YB. Usefulness of 8 $\mathrm{kDa}$ protein of Fasciola hepatica in diagnosis of fascioliasis. Korean J Parasitol. 2003; 41(2): 121123. DOI: $10.3347 / \mathrm{kjp} .2003 .41 .2 .121$.

19. Silva M, Gorman T, Alcaíno H. Inmunodiagnóstico de fasciolosis humana y ovina empleando una fracción de 24-29 kDa de F. hepatica obtenida mediante inmunoadsorción. Parasitol Latinoam. 2005; 60(1-2): 38-42. DOI: $10.4067 / \mathrm{S} 0717-$ 77122005000100005.

20. Lehner RP, Sewell MMH. A study of the antigen produced by adult Fasciola hepatica maintained in vitro. Paras Immunol. 1980; 2(2): 99-109. DOI: 10.1111/j.1365-3024.1980.tb00046.x.

21. Cornejo H, Oblitas F, Cruzado S, Quispe W. Evaluación de una prueba de ELISA con antígeno metabólico de Fasciola hepatica para el diagnóstico de fasciolosis humana en Cajamarca, Perú. Rev Peru Med Exp Salud Pública. 2010; 27(4): 569-574. DOI: $10.1590 /$ S1726-46342010000400012.

22. Colmenares C, Méndez L, Díaz-Bello Z, Alarcón B. Antígeno excreción-secreción de Fasciola hepatica: ultrafiltración y aplicación en inmunodiagnóstico. Acta Bioquím Clín Latinoam. 2007; 41(2): 259-266.

23. Voller A, Bartlett A, Bidwell DE. Enzyme immunoassays for parasitic diseases. Tras Roy Soc Trop Med Hyg. 1976; 70(2): 98-106. DOI:10.1016/0035-9203(76)90163-2.

24. Salimi-Bejestani MR, McGarry JW, Felstead S, 
Ortiz P, Akca A, Williams DJ. Development of an antibody-detection ELISA for Fasciola hepatica and its evaluation against a commercially available test. Res Vet Sci. 2005; 78(2): 177-181. DOI: 10.1016/j. rvsc.2004.08.005.

25. Orozco LC, Ovalle CE, Duque S, López MC, Nicholls RS. Exactitud del ELISA para el diagnóstico de fascioliasis bovina: análisis del área bajo la curva del receptor operador (ROC). Biomédica. 1996; 16(2): 122-130. DOI: 10.7705/ biomedica.v16i2.902.

26. Pfister K, Daveau CH, Ambroise-Thomas P. Partial purification of somatic and excretory-secretory products of adult Fasciola hepatica and their application for the serodiagnosis of experimental and natural fascioliasis using on ELISA. Res Vet Sci. 1984; 37(1): 39-43.

27. Espino AM, Diaz A, Perez A, Finlay CM. Dynamics of antigenemia and coproantigens during a human Fasciola hepatica outbreak. J Clin Microb. 1998; 36(9): 2723-2726.

28. Wilches C, Jaramillo J, Muñoz D, Robledo S, Velez I. Presence of infection by Fasciola hepatica in inhabitants from the valle de San Nicolás, eastern Antioquia. Infectio. 2009; 13(2): 92-99. DOI: 10.1016/S0123-9392(09)70730-1. 